\title{
Statistical Density Estimation Using Threshold Dynamics for Geometric Motion
}

\author{
Tijana Kostić • Andrea Bertozzi
}

Received: 21 December 2011 / Revised: 21 May 2012 / Accepted: 30 May 2012 /

Published online: 12 June 2012

(C) The Author(s) 2012. This article is published with open access at Springerlink.com

\begin{abstract}
Our goal is to estimate a probability density based on discrete point data via segmentation techniques. Since point data may represent certain activities, such as crime, our method can be successfully used for detecting regions of high activity. In this work we design a binary segmentation version of the well-known Maximum Penalized Likelihood Estimation (MPLE) model, as well as a minimization algorithm based on thresholding dynamics originally proposed by Merriman et al. (The Computational Crystal Growers, pp. 73-83, 1992). We also present some computational examples, including one with actual residential burglary data from the San Fernando Valley.
\end{abstract}

Keywords Statistical density estimation - Image segmentation · Thresholding · Ginzburg-Landau functional

\section{Introduction}

The idea to use binary segmentation techniques for density estimates arises from the need to quickly and accurately locate the regions of higher activity, as well as to calculate the local density. Instead of obtaining a complete density function estimate, such as seen in [22] and [28], our goal is to segment only one region at a time. Maximum penalized likelihood estimation models have become standard for estimating a probability density function $d(\mathbf{x})$ based on point data $\mathbf{x}_{1}, \mathbf{x}_{2}, \ldots, \mathbf{x}_{N} \in \mathbb{R}^{n}$, and they take a form

$$
\hat{d}(\mathbf{x})=\arg \max _{\substack{d(\mathbf{x}) \geq 0 \\ d=1}}\left\{\sum_{i=1}^{N} \log \left(d\left(\mathbf{x}_{i}\right)\right)-\alpha R(d)\right\}
$$

T. Kostić · A. Bertozzi (凶)

Mathematics Department, UCLA, Box 951555, Los Angeles, CA 90095-1555, USA

e-mail: bertozzi@math.ucla.edu

T. Kostić

e-mail: kostict@math.ucla.edu 
where $R(d)$ is a penalty function. A wide variety of different penalty functions are proposed in the literature, and many of them are designed to impose additional smoothness on the density function. Single variable density estimation with a non-smooth density function is discussed in [27] and [18] where the authors of both works introduced the TV semi-norm as a penalty function. In their work [22], Mohler et al. construct the first two dimensional TV norm based MPLE:

$$
\hat{d}(\mathbf{x})=\arg \max _{\substack{d(\mathbf{x}) \geq 0 \\ \int d=1}}\left\{\sum_{i=1}^{N} \log \left(d\left(\mathbf{x}_{i}\right)\right)-\alpha \int|\nabla d|\right\} .
$$

The authors also create an efficient computational method for minimization of (2) based on the Split Bregman algorithm [15]. Smith et al. in [28] propose a modification of the model (2), as well as the model that uses the $H^{1}$ norm as a penalty function, and they successfully integrated geographic information of the observed region to get a more accurate density estimate. The authors use city maps, census data as well as other types of geographical data to determine the region where the events typically occur, the valid region $D$. Knowing that the density function is zero anywhere outside the valid region, the authors align the zero level sets of the valid region and the density function. In their modified MPLE model aligning is achieved through addition of the alignment term, and the model they propose is

$$
\hat{d}(\mathbf{x})=\arg \max _{\substack{d(\mathbf{x}) \geq 0 \\ d d=1}}\left\{\sum_{i=1}^{N} \log \left(d\left(\mathbf{x}_{i}\right)\right)+\lambda \int d \nabla \cdot \frac{\nabla 1_{D}}{\left|\nabla 1_{D}\right|}-\alpha \int|\nabla d|\right\}
$$

where $1_{D}$ is a characteristic function of the valid region. In the case of the weighted $H^{1}$ MPLE model, in order to allow the density function $d$ to have sharp jumps on the border of the valid region, the $H^{1}$ penalty functional was forced away from the boundary of the valid region. Thus, the proposed model becomes:

$$
\hat{d}(\mathbf{x})=\arg \max _{\substack{d(\mathbf{x}) \geq 0 \\ \int d=1}}\left\{\sum_{i=1}^{N} \log \left(d\left(\mathbf{x}_{i}\right)\right)-\alpha \int z_{\epsilon}^{2}|\nabla d|^{2}\right\},
$$

where $z_{\epsilon}$ is a continuous function such that

$$
z_{\epsilon}= \begin{cases}1 & \text { if distance }(\mathbf{x}, \partial D)>\epsilon \\ 0 & \text { if } \mathbf{x} \in \partial D\end{cases}
$$

In this work we propose the variational MPLE segmentation model using the GinzburgLandau functional as a regularizer to segment the geography and calculate the density function:

$$
\arg \min _{u(x)} \int \epsilon|\nabla u|^{2}+\frac{1}{\epsilon} W(u) d x-\mu \int w \log (d(u)) d x
$$

where $w$ is a function that represents the given data, $u$ is a segmentation function, and $d(u)$ is a two phase density function that can be determined from $u$. We define $w$ as a sum of Dirac $\delta$ functions $w=\sum_{i}^{N} \delta\left(\mathbf{x}_{i}\right)$, where $\mathbf{x}_{i}$ are data points. This function is introduced for the purpose of more compact notation. Note that in Eq. (5) the maximum likelihood term $\int w \log (d(u)) d x$ involves the data function $w$, while in Eqs. (4), (3), (2) and (1) we used 
the sum $\sum_{i=1}^{N} \log \left(d\left(\mathbf{x}_{i}\right)\right)$. The dense region $\Sigma$ is located via segmentation function $u$, i.e. $u=\chi_{\Sigma}$. Using the information on the data set we obtain through function $w$, as well as the assumption that density $d(u)$ is piecewise constant, we calculate the values of $d(u)$ in $\Sigma$ and $\Sigma^{C}$.

This paper is organized as follows, in Sect. 2 we give some background on variational methods for image segmentation, in Sect. 3 we describe the MBO scheme, in Sect. 4 we present some background on MPLE models. In Sect. 5 we discuss the proposed model in more details, and calculate the time dependent Euler-Lagrange equation to minimize the functional (5). Section 6 explains the thresholding dynamics for minimization of our energy functional. Details on the numerical implementation are presented in Sect. 7.

\section{Background on Variational Methods in Image Segmentation and MBO Scheme}

Variational segmentation models are widely successful in image processing. In their pioneering work [23], Mumford and Shah propose the following segmentation model:

$$
\min _{\substack{K \subseteq D \\ u: D \rightarrow \mathbf{R}}} M S(u, K)=\int_{D \backslash K}|\nabla u|^{2} d x+\mu \operatorname{Length}(K)+\lambda \int_{D}(u-f)^{2} d x
$$

where $f$ is an image that should be segmented, and $u$ is a segmentation function. One of the major results in image segmentation is Chan-Vese algorithm presented in [5], where authors, inspired by the level set method by Osher and Sethian [24], propose a method for minimizing the piecewise constant version of (6):

$$
\min _{\substack{\Sigma \subseteq D \\ c_{1}, c_{2} \in \mathbf{R}}} E\left(\Sigma, c_{1}, c_{2}\right)=\operatorname{Per}(\Sigma ; D)+\lambda \int_{\Sigma}\left(c_{1}-f\right)^{2} d x+\lambda \int_{D \backslash \Sigma}\left(c_{2}-f\right)^{2} d x
$$

where $\Sigma$ is a segmented region. The level set version of (7) proposed in [5]

$$
\min _{\substack{\phi: D \rightarrow \mathbf{R} \\ c_{1}, c_{2} \in \mathbf{R}}} \int_{D}|\nabla H(\phi)|+\lambda\left\{H(\phi)\left(c_{1}-f\right)^{2}+(1-H(\phi))\left(c_{2}-f\right)^{2}\right\} d x
$$

is obtained by the boundary of $\Sigma$ being represented as a 0 -level set of the function $\phi(x)$ : $D \rightarrow \mathbf{R}$. After the optimal values for constants $c_{1}$ and $c_{2}$ are determined, the time-dependent Euler-Lagrange equation for $\phi$ is found:

$$
\phi_{t}=H_{\epsilon}^{\prime}(\phi)\left\{\nabla \cdot\left(\frac{\nabla \phi}{|\nabla \phi|}\right)-\lambda\left\{\left(c_{1}-f\right)^{2}-\left(c_{2}-f\right)^{2}\right\}\right\}
$$

where $H_{\epsilon}$ is an approximation of the Heaviside function, and a semi-implicit numerical scheme is created to solve (9). Due to the computational complexity of the numerical algorithm that solves Eq. (9), Gibou and Fedkiw created algorithms that do not explicitly solve (9). In their work [16], they designed the hybrid k-means level set method and applied it to images that were previously processed using the Perona-Malik diffusion. Another algorithm that successfully minimizes the piecewise constant Mumford-Shah functional without solving the gradient descent equation (9) is proposed in [30]. Numerous modifications of the piecewise constant Mumford-Shah model, and different ways to minimize it appeared in the literature (see [2, 4, 6, 9, 14, 17] and [29]). 
The regularization based on the Total Variation semi-norm (TV) is widely used and established as a standard procedure in numerous image processing applications. It has been proven in [19] that the Ginzburg-Landau functional converges to the TV semi-norm in the sense of $\Gamma$-convergence. This property justifies the substitution of the TV norm for the Ginzburg-Landau functional. The reason one might prefer the GL functional over the TV semi-norm is the simplicity of the minimization numerical scheme that the GL functional yields. Indeed, the $L^{2}$ gradient descent of the GL functional gives us the Allen-Cahn equation

$$
u_{t}=2 \epsilon \Delta u-\frac{1}{\epsilon} W^{\prime}(u)
$$

as opposed to having a nonlinear curvature term obtained through minimization of the TV semi-norm, although the Split Bregman technique [15] successfully resolves that issue. Models with the GL functional are typically referred to as diffuse interface models, because the double well potential causes the minimizer of the functional to have two phases with a diffuse interface between them. The diffuse interface scale is $O(\epsilon)$. The Ginzburg-Landau functional has appeared in many image processing applications, such as inpainting $[3,7]$ and segmentation $[9,10]$.

In the case of motion by mean curvature a planar curve moves with a normal velocity that is proportional to the curvature at any given point. In their work [20], Merriman, Bence and Osher introduced an intuitive and efficient level set method to approximate the motion of an interface by its mean curvature flow. The authors analyzed the motion by mean curvature of the curve $C$ by studying the diffusion equation $\chi_{t}=\Delta \chi$, where $\chi$ is the characteristic function of the set $\Sigma$ and $\partial \Sigma=C$, i.e. $C$ represents a sharp front between two phases of the characteristic function. The appropriate change of coordinates suggested in [20] reveals that, when diffusion is applied, any point on the front moves with the normal velocity that is equal to the mean curvature at that point. Simultaneously, the front is radially blurred. However, the $\chi=\frac{1}{2}$ level set is invariant to blurring. From there it follows that, the $\chi=\frac{1}{2}$ level set yields motion by mean curvature. Based on the previous observation the following numerical scheme is proposed:

- Step 1 Let $v(x)=S(\delta t) u_{n}(x)$ where $S(\delta t)$ is a propagator by time $\delta t$ of the equation:

$$
v_{t}=\Delta v
$$

with appropriate boundary conditions.

- Step 2 Threshold

$$
u_{n+1}(x)= \begin{cases}0 & \text { if } v(x) \in\left(-\infty, \frac{1}{2}\right] \\ 1 & \text { if } v(x) \in\left(\frac{1}{2}, \infty\right)\end{cases}
$$

The reason we are interested in motion by mean curvature flow is due to is its close relation to the Allen-Cahn equation (10).

It was shown in [25] that limit of the rescaled solutions $u_{\epsilon}\left(x, \frac{t}{\epsilon}\right), \epsilon \rightarrow 0^{+}$of the AllenCahn equation yields motion by mean curvature. The modification of the MBO scheme proposed in [9] by Esedoglu and Tsai particularly interesting. In their paper [9], the authors proposed a diffuse interface version of (7)

$$
M S_{\epsilon}\left(u, c_{1}, c_{2}\right)=\int_{D} \epsilon|\nabla u|^{2}+\frac{1}{\epsilon} W(u)+\lambda\left\{u^{2}\left(c_{1}-f\right)^{2}+(1-u)^{2}\left(c_{2}-f\right)^{2}\right\} d x
$$


for segmenting the image $f$. The first variation of the model (11) yields the following gradient descent equation:

$$
u_{t}=2 \epsilon \Delta u-\frac{1}{\epsilon} W^{\prime}(u)+2 \lambda\left\{u\left(c_{1}-f\right)^{2}+(1-u)\left(c_{2}-f\right)^{2}\right\}
$$

and the adaptation of the MBO scheme was used to solve it. Similarly to the MBO scheme where the propagation step based on the heat equation is combined with thresholding, Esedoglu and Tsai proposed the following scheme:

- Step 1 Let $v(x)=S(\delta t) u_{n}(x)$ where $S(\delta t)$ is a propagator by time $\delta t$ of the equation:

$$
w_{t}=\Delta w-2 \tilde{\lambda}\left(w\left(c_{1}-f\right)^{2}+(1-w)\left(c_{2}-f\right)^{2}\right)
$$

with appropriate boundary conditions.

- Step 2 Set

$$
u_{n+1}(x)= \begin{cases}0 & \text { if } v(x) \in\left(-\infty, \frac{1}{2}\right] \\ 1 & \text { if } v(x) \in\left(\frac{1}{2}, \infty\right)\end{cases}
$$

Based on the computational experiments, the authors presented their conclusions about the choice of $\delta t$ timestep in the step 1. of the algorithm. If $\delta t$ is chosen too large comparing to the parameter $\lambda^{-1}$, the interface tend to be overly smooth. As the advantage of choosing larger values for $\delta t$ they mention faster convergence. The segmentation could also benefit from the larger values for the parameter $\lambda$, as there is less penalty on high curvature of the interface in this case. The size of the spatial resolution has to be taken into account when the value for $\delta t$ is chosen, as the values much smaller than the spatial resolution could lead to the stillness of interface. These observations can also be used as guidance in parameter selection for our algorithm. The authors also show that MBO thresholding type methods for binary segmentation can easily be generalized to multi-phase segmentation methods. To accomplish that, the authors propose the four phase model based on the modified version of (11) with the sum of the two Ginzburg-Landau functionals built around two different segmentation functions, $u_{1}$ and $u_{2}$. In this case, the fidelity term naturally depends on both $u_{1}$ and $u_{2}$. After they find the gradient descent equations with respect to $u_{1}$ and $u_{2}$, they construct the thresholding numerical scheme to solve the obtained system of parabolic equations. We will adapt ideas by Esedoglu and Tsai to solve our MPLE problem and illustrate the usefulness of this simple method.Some extension of the MBO algorithms appeared in [11, 12, 21] An efficient algorithm for motion by mean curvature using adaptive grids was proposed in [26].

\section{MPLE Methods and Proposed Model}

Let us assume our data consists of $n$ points $\mathbf{x}_{1}, \mathbf{x}_{2}, \ldots, \mathbf{x}_{n}$ and is a sample of $n$ independent random variables with common density $d_{0}$. Maximum likelihood estimation is a standard method for estimating a density function $d_{0}$ based on given data. In the case the of the parametric model, we know that $d_{0}$ belongs to the family of density functions $D=\{d(\cdot, \theta): \theta \in \Theta\}$, and our goal is to find a parameter $\theta_{0}$ such that $d\left(\cdot, \theta_{0}\right)=d_{0}(\cdot)$. This class of problems are known as parametric density estimates. In 1922, According to Fisher's model [13], an optimal parameter $\theta_{0} \in \Theta$ (where $\Theta$ is a set of all parameters) satisfies the following:

$$
\theta_{0}=-\min _{\theta \in \Theta} \frac{1}{n} \sum_{i=1}^{n} \log d\left(\mathbf{x}_{i}, \theta\right) .
$$


However, in many applications, a parametric model may not be available, or information on the family of density functions $d_{0}$ belongs to may be unknown, in which case we are dealing with a nonparametric density estimate. The analog of the model (13) is an ill-posed problem, i.e. finding a probability density function $d_{0}$ such that

$$
d_{0}=-\min _{\substack{d: \Omega \rightarrow \mathbb{R} \\ j d=1}} \frac{1}{n} \sum_{i=1}^{n} \log d\left(\mathbf{x}_{i}\right)
$$

has no solution, and thus can not be directly applied. To avoid "rough" density functions, with large values concentrated around data points and small values elsewhere, the roughness penalization function $R(d)$ was introduced, and this method is known as maximum penalized likelihood estimation:

$$
d(\mathbf{x})=\max _{\substack{d: \Omega \rightarrow \mathbb{R} \\ \int d=1}}\left\{\sum_{i=1}^{N} \log \left(d\left(\mathbf{x}_{i}\right)\right)-\alpha R(d)\right\} .
$$

Different penalty functionals appeared in literature, such as $R(d)=\int_{\Omega}|\nabla \sqrt{d}|^{2}$, or $R(d)=$ $\int_{\Omega}\left|\nabla^{3} \log d\right|^{2}$ from [8]. These, and many other standard penalty functional enforce smoothness on density function, but do not perform well when the density function has sharp gradients, i.e. is piecewise constant. To resolve this issue, Koenker and Mizera in [18] as well as Sardy and Tseng in [27] propose the penalty functional to be the TV semi-norm. This approach was also successfully used in [28] and [22]. In our work, since we assume the density is a step function, choosing a penalty functional that can successfully handle sharp gradients is crucial. As previously mentioned, instead of the TV semi-norm, we chose the Ginzburg-Landau functional to be the penalty functional.

\subsection{General Model}

For now are going to focus on the segmentation function $u$. We assume our segmentation function is the characteristic function of the region $\Sigma$, where $\Sigma$ is an area with a larger density. For any given data and any given segmentation function there is a unique density function corresponding to them. With $w$ being the function that approximates the data, the total number of events is approximately equal to $\int w$, while the number of events inside and the number of events outside of the region $\Sigma$ are approximated by $\int w u$ and $\int w(1-u)$, respectively. According to that, the density $c_{1}(u)$ inside the region $\Sigma$ is equal to $\frac{\int w u}{\int u \int w}$ and the density $c_{2}(u)$ in the region $\Sigma^{C}$ is equal to $\frac{\int w(1-u)}{\int w \int(1-u)}$. Finally, we write the density function as $c_{1}(u) u+c_{2}(u)(1-u)$. The established correspondence between the segmentation and the density function suggests that building a diffuse interface MPLE model around the segmentation function is possible. As the segmentation function takes only 0 and 1 values, the Ginzburg-Landau functional is a natural choice. As the density is a rescaled segmentation function, using the Ginzburg-Landau functional for $u$, as opposed to the Ginzburg-Landau functional for the density seems both reasonable and convenient.

Here we propose the diffuse interface Maximum Penalized Likelihood Estimation model.

$$
\arg \min _{u(x)} \int \epsilon|\nabla u|^{2}+\frac{1}{\epsilon} W(u) d x-\mu \int w \log \left(c_{1}(u) u+c_{2}(u)(1-u)\right) d x
$$


where $c_{1}(u)=\frac{\int w u}{\int u \int w}$ and $c_{2}(u)=\frac{\int w(1-u)}{\int w \int(1-u)}, w$ represents the given data, $W(u)=u^{2}(1-u)^{2}$ and $\mu$ is a parameter. As we already mentioned, the Ginzburg-Landau functional converges to the TV norm in the sense of $\Gamma$ convergence, as $\epsilon \rightarrow 0^{+}$. As a consequence, the diffuse interface model we propose here converges to the TV-based MPLE that was used in [22, 28 ], when $\epsilon \rightarrow 0^{+}$. Now, variation of energy of (16) gives us the following cases for the $L^{2}$ gradient descent equation:

- If both and $c_{1}(u)$ and $c_{2}(u)$ (further we use $c_{1}$ and $c_{2}$ instead for simplicity of the notation) are non-zero:

$$
u_{t}=2 \epsilon \Delta u-\frac{1}{\epsilon} W^{\prime}(u)+\mu w\left[\frac{c_{1}-c_{2}}{c_{1}} u+\frac{c_{1}-c_{2}}{c_{2}}(1-u)+\left(\frac{\int(1-u) w}{\int 1-u}-\frac{\int u w}{\int u}\right)\right] .
$$

- If $c_{1}$ is equal to zero:

$$
u_{t}=2 \epsilon \Delta u-\frac{1}{\epsilon} W^{\prime}(u)+\mu\left[w(u-1)+\left(\frac{\int(1-u) w}{\int 1-u}-w\right)\right] .
$$

- If $c_{2}$ is equal to zero

$$
u_{t}=2 \epsilon \Delta u-\frac{1}{\epsilon} W^{\prime}(u)+\mu\left[w u+\left(w-\frac{\int u w}{\int u}\right)\right] .
$$

\subsection{Special Case Model}

For the special case problem the density in the region $\Sigma^{C}$ is zero, so the density function is just a rescaled segmentation function. Thus, replacing the density function $c_{1}(u) u+$ $c_{2}(u)(1-u)$ in the MPLE term of our general model by the segmentation $u$ seems reasonable. Now, the model we propose is:

$$
\arg \min _{u(x)} \int \epsilon|\nabla u|^{2}+\frac{1}{\epsilon} W(u) d x-\mu \sum_{i, j} w_{i, j} \log \left(u_{i, j}\right) .
$$

Once again, assuming $w$ is a function that approximates the data, in order to make everything well-defined we introduce the model with a small constant $v$ :

$$
\arg \min _{u(x)} \int \epsilon|\nabla u|^{2}+\frac{1}{\epsilon} W(u) d x-\mu \int w \log ((1-v) u+v(1-u)) d x .
$$

The Euler-Lagrange equation for the energy functional (21) is:

$$
u_{t}=2 \epsilon \Delta u-\frac{1}{\epsilon} W^{\prime}(u)+\mu w\left(\frac{1}{1-v} u+\frac{1}{v}(1-u)\right)(1-2 v) .
$$

\section{Proposed Dynamics}

We use the following gradient descent equation

$$
u_{t}=2 \epsilon \Delta u-\frac{1}{\epsilon} W^{\prime}(u)+A(u(\cdot, t)) u+B(u(\cdot, t))
$$


with $A(u(\cdot, t))$ and $B(u(\cdot, t))$ being non-linear functions. Each of the gradient descent equations, (17), (18), (19) and (22) can be given in the form (23), where $A(u(\cdot, t))$ and $B(u(\cdot, t))$ take different vales in different cases.

$$
\begin{aligned}
& A(u(\cdot, t))=-\mu w \frac{\left(c_{1}-c_{2}\right)^{2}}{c_{1} c_{2}} \\
& B(u(\cdot, t))=\mu w \frac{c_{1}-c_{2}}{c_{2}}+\left(c_{2}-c_{1}\right) \int w \text { in Eq. (17) } \\
& A(u(\cdot, t))=\mu w \\
& B(u(\cdot, t))=\mu\left(c_{2} \int w-2 w\right) \quad \text { in Eq. (18) } \\
& A(u(\cdot, t))=\mu w \\
& B(u(\cdot, t))=\mu\left(w-c_{1} \int w\right) \quad \text { in Eq. (19) } \\
& A(u(\cdot, t))=-\mu w \frac{(2 v-1)^{2}}{(1-v) v} \\
& B(u(\cdot, t))=\mu w \frac{1-2 v}{v} \quad \text { in Eq. (22) }
\end{aligned}
$$

Motivated by the MBO scheme for solving the Allen-Cahn equation, we propose a thresholding scheme to approximate the solution of Eq. (23). Esedoglu and Tsai used a similar approach to minimize the Mumford-Shah segmentation functional in [9]. The first step we need to take toward generating a thresholding scheme is finding a good way to split Eq. (23) into two steps analogous to those proposed in the MBO scheme. In that regard, finding a way that successfully deals with the non-linear forcing term of Eq. (23) is critical. Inspired by the algorithm presented in [9] we propose the following dynamics:

- Step 1. Let $v(x)=S(\delta t) u_{n}(x)$ where $S(\delta t)$ is a propagator by time $\delta t$ of the equation:

$$
y_{t}=\Delta y-A(y(\cdot, t)) y+B(y(\cdot, t))
$$

with appropriate boundary conditions.

- Step 2. Set

$$
u_{n+1}(x)= \begin{cases}0 & \text { if } v(x) \in\left(-\infty, \frac{1}{2}\right] \\ 1 & \text { if } v(x) \in\left(\frac{1}{2}, \infty\right)\end{cases}
$$

\section{Numerical Implementation}

In the propagation phase of the algorithm we solve the following PDE:

$$
v_{t}=\Delta v-A(v(\cdot, t)) v+B(v(\cdot, t))
$$

with the $u_{n}$ being an initial condition. To generate the numerical results we denote the timestep by $\delta \tau$ is a timestep, and approximate the Laplacian by its five-point stencil, which 
gives us the following scheme:

$$
\frac{v^{n+1}-v^{n}}{\delta \tau}=\Delta v^{n+1}+A\left(v^{n}(\cdot, t)\right) v^{n}+B\left(v^{n}(\cdot, t)\right) .
$$

As the linear system described above is circulant we find the Fast Fourier transform (FFT) a convenient way solve it. We are aware that other solvers with even better computational complexity than the FFT, such as multigrid algorithm, could be used. In the propagation phase our goal is not to achieve steady state, and iterations are repeated only until the total propagation time reached $\delta t$. After the propagation phase, a thresholding step is necessary to complete the iteration:

$$
u_{i, j}^{n+1}= \begin{cases}0 & \text { if } v_{i, j}^{l} \in\left(-\infty, \frac{1}{2}\right] \\ 1 & \text { if } v_{i, j}^{l} \in\left(\frac{1}{2}, \infty\right)\end{cases}
$$

where $l$ is a total number of iterations we made in the propagation phase. The small relative change of the $L^{2}$ norm between two consecutive iterations was used as a stopping criterion.

In this implementation, the data function $w$ is used as an initial condition, along with Dirichlet or Neumann boundary conditions.

\subsection{Adaptive Timestepping}

The choice of timestep in the propagation phase, a "sub-timestep", can be chosen to optimize performance. In the early stage of computation, it is important to keep the sub-timestep small in order to obtain a good estimate in the propagation phase. However, as our algorithm is approaching steady state, a large number of iterations in the propagation phase pose a burden on the computational time. To successfully speed up the convergence of our algorithm, we used adaptive timestepping, a modified form of the scheme proposed in [1].

The scheme uses a dimensionless local truncation error calculated in every iteration, and the timestep is increased when the error is smaller then a chosen tolerance. The error at time $t^{n}$ uses solution at three consecutive timesteps $t^{n-1}, t^{n}$ and $t^{n+1}$. Let us define $e^{n+1}=$ $\frac{\left(v^{n+1}-v^{n}\right)}{v^{n}}$ and $e^{n}=\frac{\left(v^{n}-v^{n-1}\right)}{v^{n}}$, as well as $\Delta t_{\text {old }}=t^{n}-t^{n-1}$. The previous definitions allow us to define a dimensionless estimate of the local truncation error:

$$
\left\|e^{n+1}-\frac{\Delta t}{\Delta t_{\text {old }}} e^{n}\right\|_{L^{2}} .
$$

This algorithm is both quick and practical. Even though other more accurate ways to estimate the measure of error are available, such as step doubling, they are often much more computationally expensive.

We used adaptive timestepping at two different levels, in the propagation phase of the algorithm we adapt the sub-timestep, as well as adapting an initial sub-timestep for the future iterations. In the propagation phase of any iteration, we calculate a dimensionless truncation error estimate for different propagation times. Once an error is smaller than a given tolerance $\mathrm{Tol}_{1}$ for a certain number of the consecutive iterations, we increase the timestep by $10 \%$. We also estimate the dimensionless error in every iteration of the algorithm, and if we find an error to be smaller than $\mathrm{Tol}_{2}$ the initial sub-timestep in the propagation phase of the next iteration will be increased be $10 \%$. However, we never allow the initial sub-timestep to be larger than $\frac{1}{8}$ of the timestep. Notice that we are not adapting the timestep, the total propagation time in each iteration is the same. 


\subsection{Adaptive Resolution}

Another way to improve the computational time is to use adaptive resolution. As we mentioned before, we use the data function $w$ as an initial condition when solving the equation (23). It is reasonable to assume that the more the initial condition "resembles" the solution, the less iterations the algorithm would take to obtain the solution. The main idea is to generate a lower resolution form of the data set, then use a low resolution solution to create a good initial guess for the high resolution solution. Providing a good initial guess for the higher resolution problem is particularly useful as the iterations when the algorithm is applied to the higher resolution versions of the data set tend to be slower. In this implementation, we typically applied this procedure several times on some sparse data sets. At each step we create the coarser form of the given data set, until we reach the version of the data set that has a satisfying density. Our experiments show that data sets with the total density between 0.05 and 0.2 are optimal for this algorithm. Once a sufficiently dense low resolution version of the data set is obtained, we run our algorithm to get the low resolution solution, and start working our way up from there. The higher resolution approximation of the solution is then generated, and used as an initial condition in the next step. In the next step, we are solving the problem on the data set that has a higher resolution. It is important to mention that this process does not alter the original data set. We call this process $n$-step adaptive resolution where $n$ is the total number of times we reduced the resolution of the original data set. The number of steps, $n$, is closely related to our choice of timestep. In case we are segmenting the region of higher density in our data, we noticed, through multiple experiments, that the timestep often can be given as $\omega 2^{n}$, where $n$ is the number of levels in adaptive resolution, and $\omega \in[0.15,0.2]$. In case we are locating the valid region, we usually allow a smaller timestep, but also a larger number of levels in adaptive resolution. However, starting with a problem that has a significantly lower resolution comparing to the original one, we might run into some problems. Decreasing resolution significantly may result in a very different looking data set, thus segmentation would not perform in an expected way, i.e. this first initial guess would not be a good approximation of the solution we are trying to find.

\section{Computational Examples}

\subsection{Test Shapes}

To verify the performance of our algorithm, we constructed three examples of the probability density maps featuring three shapes and they are shown in Figs. 1(a), 2(a) and 3(a). Each of the densities were sampled, and the toy data sets are obtained, and presented in Figs. 1(b), 2(b) and 3(b) respectively. We applied the general version of our algorithm to solve these problems, and the results are shown in Figs. 1(c), 2(c) and 3(c). Adaptive timestepping was used in all of the examples with three shapes, while the adaptive resolution was not used, given the small scale of these examples.

\subsection{Orange County Coastline}

In their work [28] Smith et al. constructed several test data sets using the spatial information of the Orange County coastline obtained from Google Earth. The authors determined the boundary between the valid and the invalid region by locating the ocean, rivers, parks and other features the valid region of a residential crimes map naturally excludes. Then, inside 


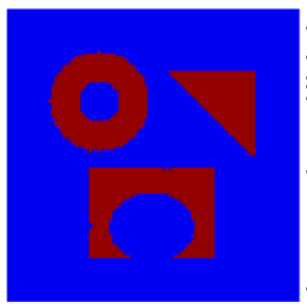

(a)

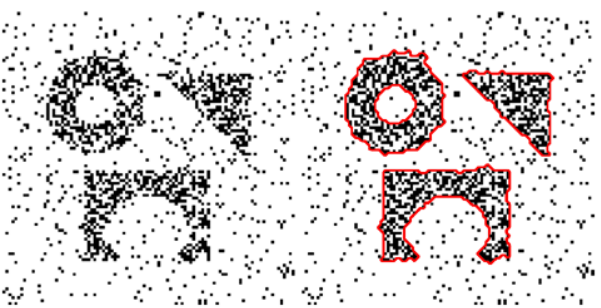

(c)

Fig. 1 Segmentation of three shapes: Image (a) presents a plot of the density map. Assuming the size of (a) is $1 \times 1$, the densities of the dense region and the sparse region are 3.3943 and 0.3927 , respectively. Image (b) is a plot of the data set, in a $100 \times 100$ pixel image, with 1449 events. Image (c) shows the contours of the segmented dense region. In the process of generating this result we used the timestep 1.6 along with the parameter $\mu=0.13$. The densities of our estimated dense and sparse regions are 3.4811 and 0.3649

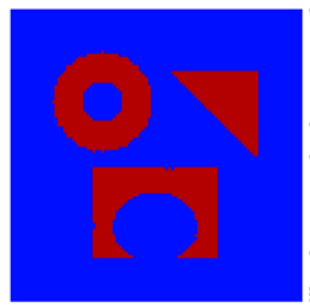

(a)

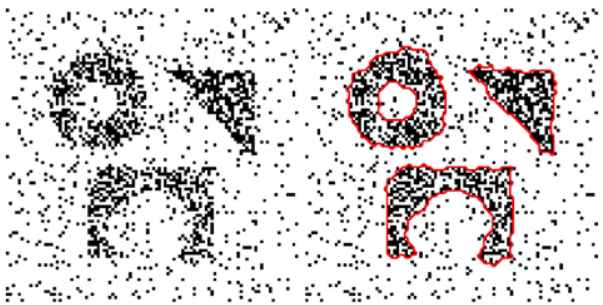

(b) (c)

Fig. 2 Segmentation of three shapes: Image (a) presents a plot of the density map. Assuming the size of (a) is $1 \times 1$, the densities of the dense region and the sparse region are 3.145 and 0.456 , respectively. Image (b) is a plot of the data set, in a $100 \times 100$ pixel image, with 1539 events. Image (c) shows the contours of the segmented dense region. The choice of parameters was 1.6 for the timestep, and $\mu=0.15$. The densities of our estimated dense and sparse regions are 3.311 and 0.422

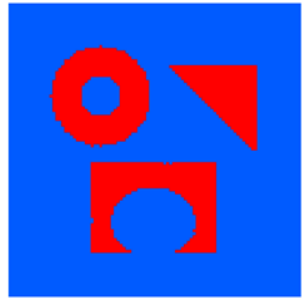

(a)

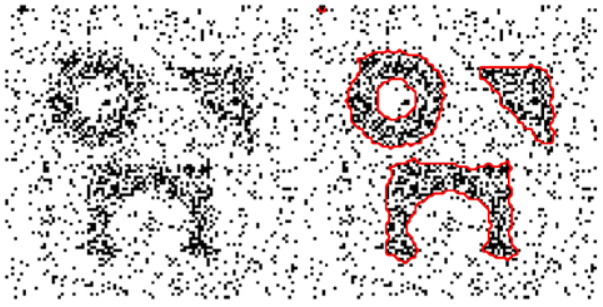

(b) (c)

Fig. 3 Segmentation of three shapes: Image (a) presents a plot of the density map. Assuming the size of (a) is $1 \times 1$, the densities of the dense region and the sparse region are 2.605 and 0.592 , respectively. Image (b) is a plot of the data set, in a $100 \times 100$ pixel image, with 1696 events. Image (c) shows the contours of the segmented dense region. The choice of parameters was 1.6 for the timestep, and $\mu=0.1$. The densities of our estimated dense and sparse regions are 2.780 and 0.573

the valid region, they constructed three other regions and assigned different densities to them, which is shown on the density map in Fig. 10, and we also, inspired by their examples, constructed the density maps shown in Fig. 4 as well as in Fig. 7. Sampling from these 
Fig. 4 The toy density map of the Orange County Coastline, featuring two disconnected dense regions

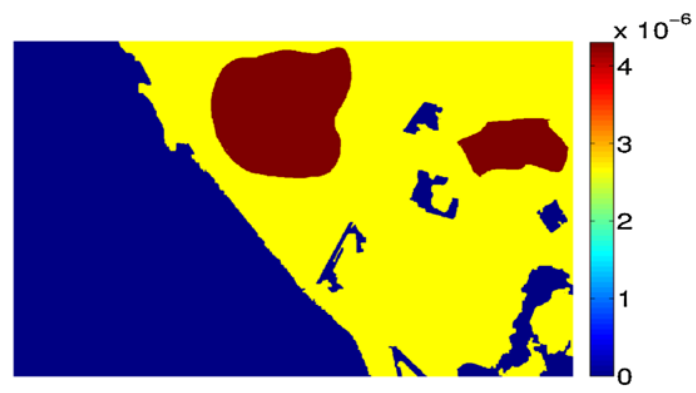

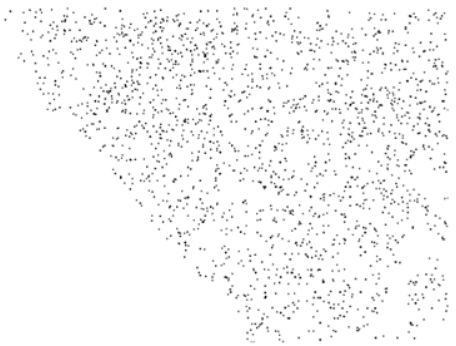

(a)

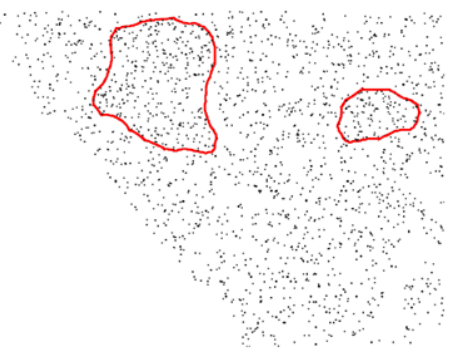

(b)

Fig. 5 A 2000 event sample of the density function from Fig. 4 is shown in (a). The contour of the segmented dense region is shown in (b). The choice of parameters is: $\mu=0.1$, timestep is 13.0 with the 3 -step adaptive resolution. The data points in this figure are manually enhanced for the purpose of more clear display of the image

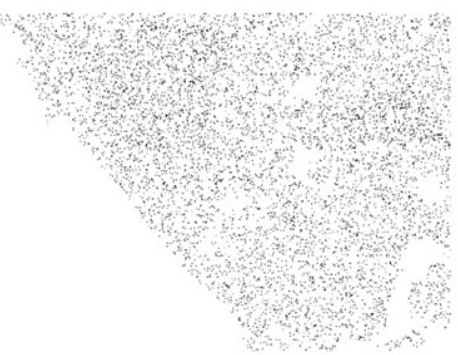

(a)

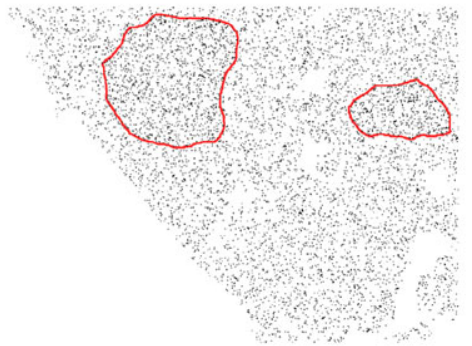

(b)

Fig. 6 An 8000 event sample of the density function from Fig. 4 is shown in (a). The contour of the segmented dense region is shown in (b). The choice of parameters is: $\mu=0.092$, timestep is 8.0 with the 2-step adaptive resolution. The data points in this figure are manually enhanced for the purpose of more clear display of the image

density functions we generated data sets shown in Figs. 5(a), 6(a), 8(a), 9(a) and 11(a). A segmentation of the corresponding dense region is shown next to each data set. Note that there are four different levels of densities in Fig. 10. The solution in Fig. 11 shows that the different choice of the parameter $\mu$ can lead to segmentation of the dense regions at different levels. All images in this section have resolution of $600 \times 1000$ pixels. 
Fig. 7 The toy density map of the Orange County Coastline, featuring three disconnected dense regions

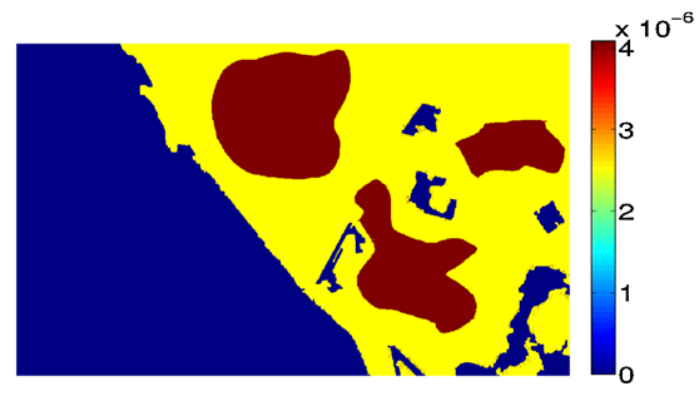

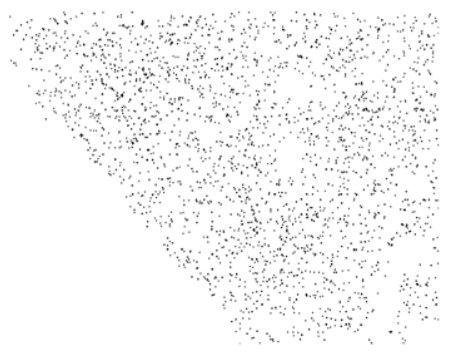

(a)

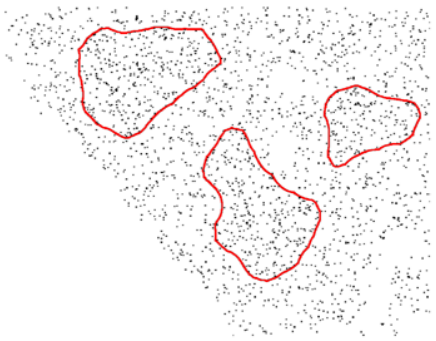

(b)

Fig. 8 A 2500 event sample of the density function from Fig. 7 is shown in (a). The contour of the segmented dense region is shown in (b). The choice of parameters is: $\mu=0.11$, timestep is 11.0 with the 3 -step adaptive resolution. The data points in this figure are manually enhanced for the purpose of more clear display of the image

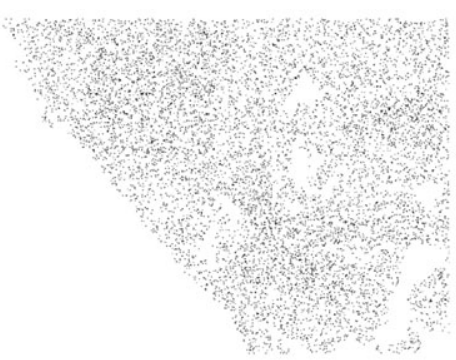

(a)

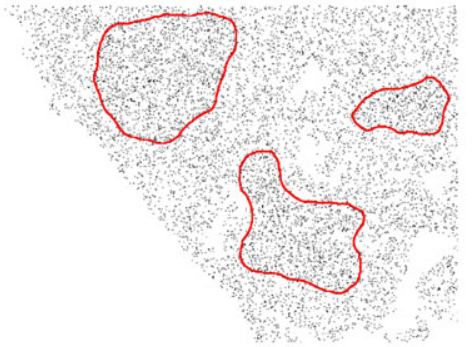

(b)

Fig. 9 A 10000 event sample of the density function from Fig. 7 is shown in (a). The contour of the segmented dense region is shown in (b). The choice of parameters is: $\mu=0.075$, timestep is 7.5 with the 2-step adaptive resolution. The data points in this figure are manually enhanced for the purpose of more clear display of the image

\subsection{San Fernando Valley Residential Burglary Data}

We may also consider a special case of our problem, the case when we assume all events are located inside the region $\Sigma$, and our goal is to segment the region. The region $\Sigma$ would represent a valid region of the given data set. In absence of geographic data that describe the location of the valid region, an accurate estimate of it can dramatically improve accuracy of the density estimation, see [28] and [22]. In the following example, our goal was to, 
Fig. 10 The toy density map of the Orange County Coastline, featuring two disconnected dense regions, and one sparse region

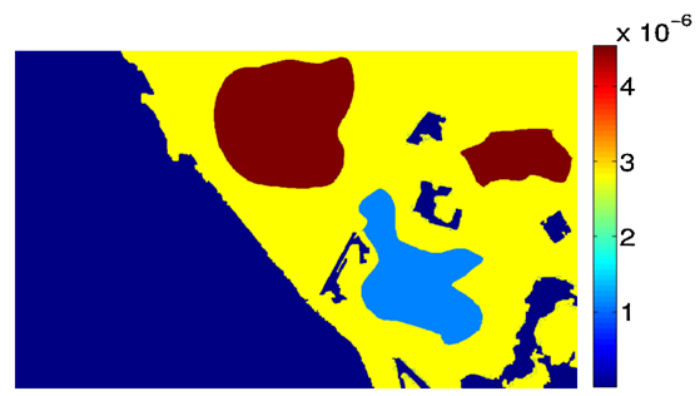

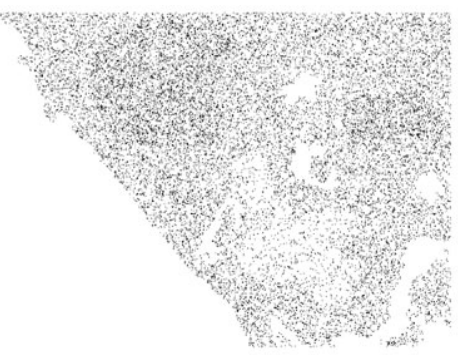

(a)

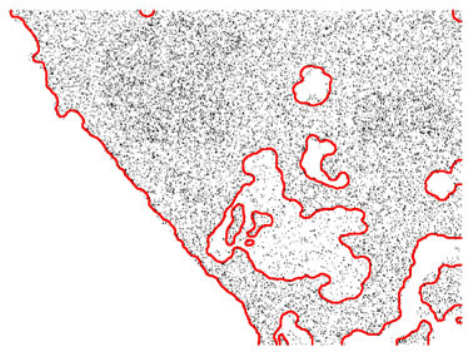

(b)

Fig. 11 A 20000 event sample of the density function from Fig. 10 is shown in (a). The contour of the segmented dense region is shown in (b). The choice of parameters is: $\mu=0.03$, timestep is 2.2 with the 2-step adaptive resolution

without using any spatial information, segment the valid region from the San Fernando Valley residential burglary data. The events in Fig. 12(a) represent locations where burglaries took place during 2004 and 2005 . The contour of our valid region estimate obtained by applying the special case model is also shown in Fig. 12(a). Smith et al. in [28] performed the valid region estimate using census and other types of data to locate the residential area in the region of interest, and their result is Fig. 12(b). They incorporated the valid region estimate from Fig. 12(b) in their Weighted $H^{1}$ MPLE model to obtain the density estimate results from Fig. 12(c). The TV MPLE algorithm developed by Mohler et al. in [22], was used to generate the density estimate in Fig. 12(b). This method did not use any additional spatial information to locate the valid region.

\section{$7 V$-Fold Cross Validation}

The choice of the smoothing parameter $\mu$ and the timestep can affect the performance of this algorithm. Our experiments show that in case we are segmenting the high density region the optimal value of the parameter $\mu$ is not larger than 0.2 . When our goal is to estimate the valid region, we typically assign larger values to the parameter $\mu$. To estimate the value of the smoothing parameter we implemented a version of the $V$-fold cross validation algorithm. In their work [27] Sardy and Tseng proposed the $V$-fold cross validation based on the Kullback-Leibler information. In the $V$-fold cross validation, the original data set is partitioned into $V$ disjoint subsets $\mathbf{x}_{v}=\left\{x_{i}, i \in S_{v}\right\}$ where $S_{v}$ consists of all indexes of data points from the partition $v=1, \ldots, V$. Set $\mathbf{x}_{-v}=\left\{x_{i}, i \notin S_{v}\right\}$ is used as a training set, 
Fig. 12 San Fernando Valley residential burglary: (a) A plot of the data set with the contours of the solution, the red contour bounds the dense region, while the blue one bounds the valid region. The size of the original data set is $605 \times 525$ pixels. We used a timestep 2.0 with $\mu=1.1$ and 3 -step adaptive resolution to find the valid region, and $\mu=0.08$, a timestep 3.5 with 1 -step adaptive resolution to locate the dense region. The data points in this figure are manually enhanced for the purpose of more clear display of the image.

(b) A valid region estimate from [28]. (c) and (d) are density estimates form [28] and [22] respectively

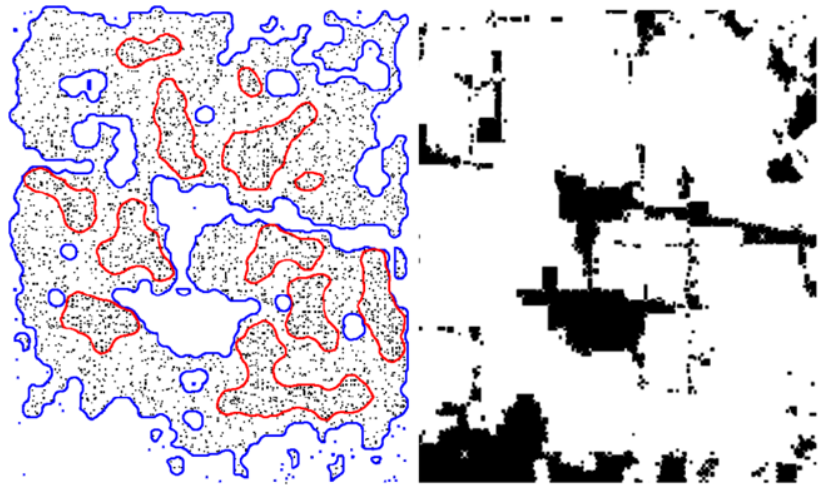

(a)

(b)

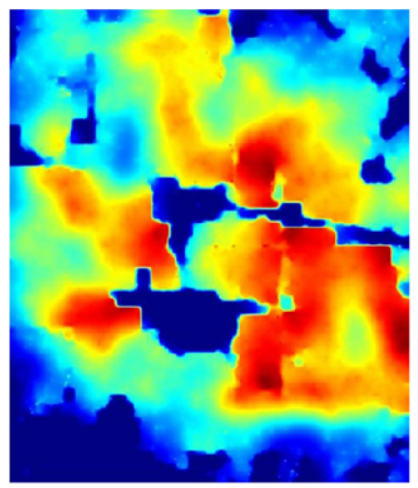

(c)

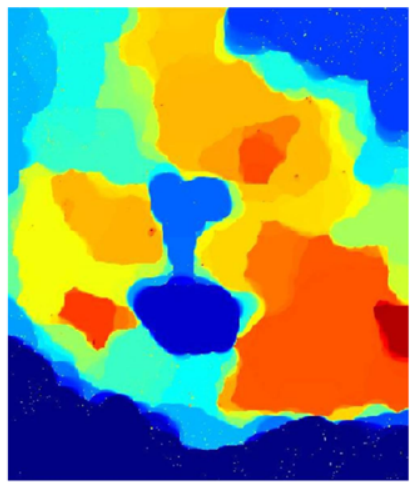

(d)

i.e. the algorithm is applied on $\mathbf{x}_{-v}$ with some particular value $\hat{\mu}$, and the density $\hat{d}_{\hat{\mu},-v}$ is estimated. Set $\mathbf{x}_{v}$ is a validating set, which means that $\left\{\hat{d}_{\hat{\mu},-v}\left(x_{i}\right)\right\}_{i \in S_{v}}$ is used to estimate the density on $\mathbf{x}_{v}$. Following these observations, the authors of [27] proposed the following estimate of Kullback-Leibler information $C V(\hat{\mu})=-\sum_{v=1}^{V} \sum_{i \in S_{v}} \hat{d}_{\hat{\mu},-v}\left(x_{i}\right)$, and after the search of the set of parameters $\hat{\mu}$ the one that minimizes this quantity is selected. However, $C V(\hat{\mu})$ uses only the log-likelihood to predict the performance of the model for some value $\hat{\mu}$ of the smoothing parameter, but does not take the $H^{1}$ norm of the segmentation function of the estimated density into account. We denote the segmentation function that corresponds to the density $\hat{d}_{\hat{\mu},-v}$ by $\hat{u}_{\hat{\mu},-v}$. Since the segmentation function is a binary function, the discrete $H^{1}$ and the discrete $T V$ norm are equivalent, thus the $H^{1}$ norm also measures the length of the front between two phases. In some applications, such as the case when the dense and the surrounding sparse region have similar densities, the values of $C V(\mu)$ for the different values of $\mu$ tend to be similar. It is useful in those cases to also measure the $H^{1}$ norm of the obtained segmentation $\hat{u}_{\hat{\mu},-v}$, and incorporate that information in the $V$-fold cross validation. Because of that, we propose a slightly different technique, where we evaluate $C V_{H^{1}}(\hat{\mu})=-\sum_{v=1}^{V}\left(\sum_{i \in S_{v}} \hat{d}_{\hat{\mu},-v}\left(x_{i}\right)-\xi \int\left|\nabla \hat{u}_{\hat{\mu},-v}\right|^{2}\right)$ for each value of $\hat{\mu}$ from some proposed set of parameters, and select the value that minimizes it. The results do not appear to be very sensitive to $\xi$, we used small values, comparable to those of $\hat{\mu}$. The evaluation of $C V_{H^{1}}(\mu)$ for a single value of parameter $\mu$ requires $V$ different density estimates, which could cause the $V$-fold cross validation to be very computationally intense. However, all 




(a)

(b)

(c)

(d)

Fig. 13 The data set shown in 3 and the results obtained using different values of $\mu$ : (a) Smoothing parameter is too small $\mu=0.12$. (b) We used $\mu$ obtained through our 10 -fold cross validation with the $H^{1}$ norm, we evaluated $C V_{H^{1}}$. (c) 10-fold cross validation from [27], $C V(\mu)$, was applied to obtain $\mu$ used in this example. (d) Smoothing parameter $\mu=0.3$, the value is larger than optimal

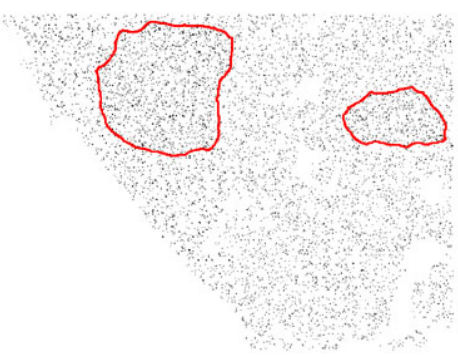

(a)

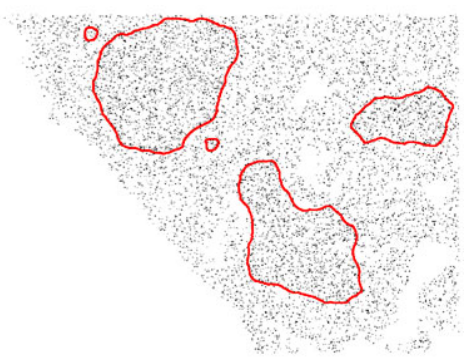

(b)

Fig. 14 10-fold cross validation was applied on the data sets displayed in Figs. 6(a) and 9(a) to select the parameter $\mu$. The data sets with the contours of the respective dense regions obtained using those values of $\mu$ are shown

density estimates that have to be performed are independent of each other, which makes the $V$-fold cross validation a perfect candidate for parallelization. In this implementation, we used 10-fold cross validation, and the process of calculating $C V_{H^{1}}$ is parallelized using 10 threads, which reduces the computational time of one evaluation of $C V_{H^{1}}$ down to the computational time needed for one density estimate. The computational time one density estimate takes varies from $0.2 \mathrm{~s}$ in the small scale examples $(100 \times 100$ pixels $)$ to around one minute in the large scale examples $(600 \times 1000$ pixels $)$. To demonstrate the performance of the proposed algorithm, Figs. 13 and 14 show some computational examples with segmentations generated using a model with the smoothing parameter obtained through 10 -fold cross validation. To find the parameter $\mu$ we performed the linear search of intervals.

\section{Conclusion}

This work demonstrates that threshold dynamics methods for image segmentation are a powerful tool for statistical density estimation for problems involving two dimensional geographic information. The efficiency of the method, especially when combined with multiresolution techniques makes this a practical choice for parameter estimation involving $V$ fold cross validation, especially when parallel platforms are available. The method is a binary segmentation method that also determines density values. However, it can be naturally generalized to multi-level segmentation. One way to achieve that may include representing 
the segmentation function as a linear combination of the multiple binary components, similarly to the idea used for generalizing binary to grayscale inpainting in [7]. However, this requires sufficient data to warrant a multi-level segmentation.

Acknowledgements This paper is dedicated to Prof. Stanley Osher on the occasion of his 70th Birthday. We would like to thank Laura Smith and George Mohler for their helpful comments. This work was supported by ONR grant N000141210040, ONR grant N000141010221, AFOSR MURI grant FA9550-10-1-0569, NSF grant DMS-0968309 and ARO grant W911NF1010472, reporting number 58344-MA.

Open Access This article is distributed under the terms of the Creative Commons Attribution License which permits any use, distribution, and reproduction in any medium, provided the original author(s) and the source are credited.

\section{References}

1. Bertozzi, A.L., Brenner, M.P., Dupont, T.F., Kadanoff, L.P.: Singularities and Similarities in Interface Flows, Trends and Perspectives in Applied Mathematics. Springer, New York (1994)

2. Brown, E.S., Chan, T.F., Bresson, X.: A convex relaxation method for a class of vector-valued minimization problems with applications to Mumford-Shah segmentation. Ucla cam report 10-43 (2010)

3. Bertozzi, A.L., Esedoglu, S., Gillette, A.: Inpainting of binary images using the Cahn-Hilliard equation. IEEE Trans. Image Process. 16(1), 285-291 (2007)

4. Chan, T.F., Nikolova, M., Esedoglu, S.: Algorithms for finding global minimizers of image segmentation and denoising models. SIAM J. Appl. Math. 66, 16-32 (2006)

5. Chan, T.F., Vese, L.A.: Active contours without edges. IEEE Trans. Image Process. 2, 266 (2001)

6. Chung, G., Vese, L.A.: Image segmentation using a multilayer level-set approach. Comput. Vis. Sci. 12(6), 267-285 (2009)

7. Dobrosotskaya, J.A., Bertozzi, A.L.: A wavelet-Laplace variational technique for image deconvolution and inpainting. IEEE Trans. Image Process. 17(5), 657-663 (2008)

8. Eggermont, P.P.B., LaRiccia, N.V.: Maximum Penalized Likelihood Estimation. Springer, Berlin (2001)

9. Esedoglu, S., Tsai, Y.R.: Threshold dynamics for the piecewise constant Mumford-Shah functional. J. Comput. Phys. 211(1), 367-384 (2006)

10. Esedoglu, S., March, R.: Segmentation with depth but without detecting junctions. J. Math. Imaging Vis. 18(1), 7-15 (2003). Special issue on imaging science, 2002

11. Esedoglu, S., Ruuth, S.J., Tsai, R.: Diffusion generated motion using signed distance functions. J. Comput. Phys. 229(4), 1017-1042 (2010)

12. Esedoglu, S., Ruuth, S.J., Tsai, R.: Threshold dynamics for high order geometric motions. Interfaces Free Bound. 10(3), 263-282 (2008)

13. Fisher, R.A.: On the mathematical foundations of theoretical statistics. Philos. Trans. R. Soc. Lond. A 222, 309-360 (1922)

14. Gao, S., Bui, T.D.: Image segmentation and selective smoothing by using Mumford-Shah model. IEEE Trans. Image Process. 14(10), 1537-1549 (2005)

15. Goldstein, T., Osher, S.: The split Bregman method for $L^{1}$-regularized problems. SIAM J. Imaging Sci. 2(2), 323-343 (2009)

16. Gibou, F., Fedkiw, R.: A fast hybrid k-means level set algorithm for segmentation. In: Proceedings of the 4th Annual Hawaii International Conference on Statistics (2005)

17. Chung, G., Vese, L.A.: Image segmentation using a multilayer level-set approach. Comput. Vis. Sci. 12(6), 267-285 (2009)

18. Koenker, R., Mizera, I.: Density estimation by total variation regularization. In: Advances in Statistical Modeling and Inference, Essays in Honor of Kjell A. Doksum, pp. 613-634. World Scientific, Singapore (2006)

19. Kohn, R.V., Sternberg, P.: Local minimisers and singular perturbations. Proc. R. Soc. Edinb. A 111(1-2), 69-84 (1989)

20. Merriman, B., Bence, J., Osher, S.: Diffusion generated motion by mean curvature. In: The Computational Crystal Growers. AMS Selection in Math., pp. 73-83 (1992)

21. Merriman, B., Ruuth, S.J.: Diffusion generated motion of curves on surfaces. J. Comput. Phys. 225(2), 2267-2282 (2007)

22. Mohler, G., Bertozzi, A., Goldstein, T., Osher, S.: Fast TV regularization for 2D maximum penalized likelihood estimation. J. Comput. Graph. Stat. 20(2), 479 (2011) 
23. Mumford, D., Shah, J.: Optimal approximations by piecewise smooth functions and associated variational problems. Commun. Pure Appl. Math. 42, 577-658 (1989)

24. Osher, S., Sethian, J.A.: Fronts propagating with curvature-dependent speed: algorithms based on Hamilton-Jacobi formulations. J. Comput. Phys. 79, 12-49 (1988)

25. Rubinstein, J., Sternberg, P., Keller, J.B.: Fast reaction, slow diffusion, and curve shortening. SIAM J. Appl. Math. 49(1), 116-133 (1989)

26. Ruuth, S.J.: Efficient algorithms for diffusion-generated motion by mean curvature. J. Comput. Phys. 144, 603-625 (1998)

27. Sardy, S., Tseng, P.: Density estimation by total variation penalized likelihood driven by the sparsity 11 information criterion. Scand. J. Stat. 37(2), 321-337 (2010)

28. Smith, L., Keegan, M., Wittman, T., Mohler, G., Bertozzi, A.: Improving density estimation by incorporating spatial information. URASIP J. Adv. Signal Process. 2010

29. Vese, L.A., Chan, T.F.: A multiphase level set framework for image segmentation using the mumford and shah model. Int. J. Comput. Vis. 50(3), 271-293 (2002)

30. Song, B., Chan, T.: A fast algorithm for level set based optimization. UCLA 